\title{
The biology of ageing
}

What causes ageing? Is it inevitable? Could it be slowed or even reversed? Human have wanted to know the ever since we became aware of our own mortality. Despite the tremendous advances that have been made in our scientific century, ageing remains one of the greatest mysteries in biological science. But scientists, like Dr Nan-ping Weng of the National Institutes of Health are slowly uncovering its secrets.

r Nan-ping Weng thinks the
best experimental approach to
study human ageing is through a gitudinal approach. A 'cross-sectiona' approach comparing groups of young individuals to older groups is commonly used and yielded rich information regarding the difference between the young and old groups. But this approach has issues, according to Weng: Substantial differences in environmen oxp subjest ist betwen young and distinguish those true biological changes with age, from different life experiences". With this in mind he tillises an approach that tracks the changes that occur to

mpacts their susceptibility to disease. In Dr Weng's group's 2016 study published in Immunity \& Ageing, Dr Firstly, was there a change in the differen types of immune cells in individuals as hey age and, secondly, could a pattern be determined by comparing individuals? To do this, they looked at four different ypes of immune cells: B cells (cells th Natural killer (NK) cells (pat of the inbut

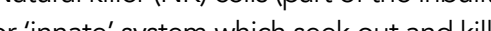
infected cells and two types of Tcells (port of the immune system that can adapt following infection. T cells come in two

Humans have wanted to know the answer to these questions ever since we became aware of our own mortality.

people over time. These follow-ups can come after 5 years, 10 years, 20 years, 0 even lifetime long. Although more timeconsuming and expensive than the crosssectional approach, so-called longitudinal studies allow researchers to minimise the their conclusions.

\section{IMMUNE SYSTEM DECLINE}

It is well known that as people age, they other diseases as well, it is one of the reasons we vaccinate the of the changes in the immune system as people (1) age, and how this

flavours, $C D 4^{+}$cells secrete signals to aid other immune cells in the fight, and CD8, which, like NK cells, seek out and kill infected cells specifically. These cells are known collectively as lymphocytes.

DrWeng and colleagues tracked the chanjects The results showed that a large degree of variability from person to person. Some showed a decline, some no change and some had an increase.

To try and understand this further, the group looked to see if the rate of change varied as people got older. They were stayed quite consistent over time.

This means that although two people

could display very different changes to their immune system as they age, the individual pattern of change did not seem to vary over time.

Interestingly, different immune cells seem to vary to different extents. Most variable

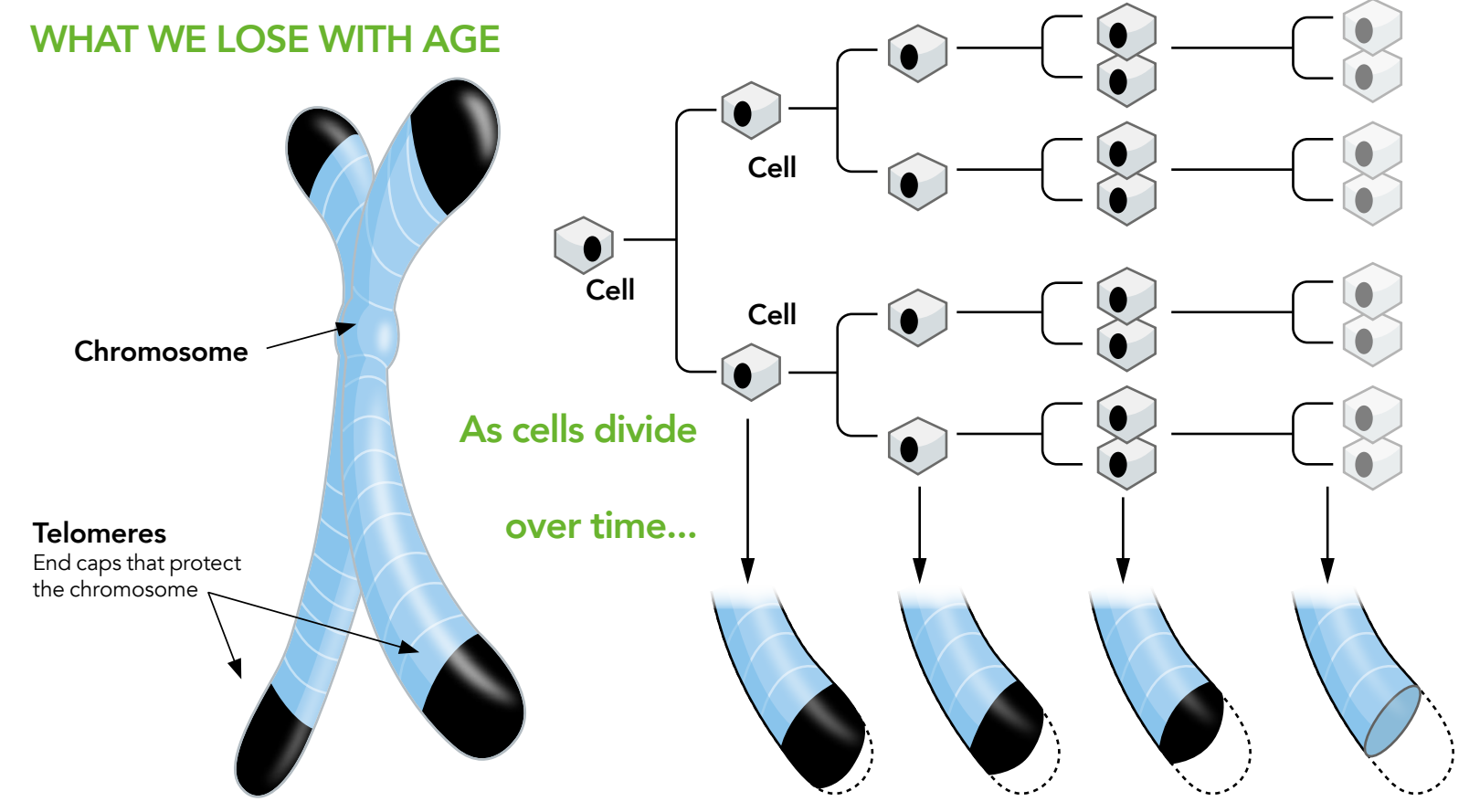

...telomeres shorten, and eventually cell division stops

were $\mathrm{CD} 4^{+} T$ cells, followed by: NK cells, $C D 8^{+} T$ cells and $B$ cells. The group wondered if these differences might be due to infection with viruses such as not to be the case. However, they did find that levels of $T$ cells correlated with levels of the cytokine (a soluble signal that controls immune function) IL-15, which is known to cause these cells to expand in number. Similarly, the number of $B$ cells was found to correlate with signal TNF-R which causes a similar expansion in these cells. Therefore, differences in the levels of these soluble signals may have produced this variability

\section{TELOMERES AND AGEING}

But what might cause the levels of for why the levels of certain immunation drop in some people as they age may lay in something called a telomere.

Each time a cell divides in two, the cell needs to make an identical duplicate of its DNA (the template that instructs a cell how to function) so that each daughter cell receives a copy. On the end of each DNA strand is a sequence called a telomere. Each time the cell divides, the telomere shortens unti eventually, it becomes so short that the is capped with thes is capped with these telomere sequences forward the idea that they may act as a safety mechanism to prevent cells from dividing out of control, which could lead to cancer

In order to fulfil their function, the lymphocytes of the immune system are required to divide many times. This has led scientists to wonder if they could be susceptible to telomere shortening. To address this question DrWeng published two studies in the journal Clinical Science (2015) and Frontiers of Immunology of immune cell telomeres as people age.

The first study was performed in 216 people aged between $20-90$ years of and the second study was performed

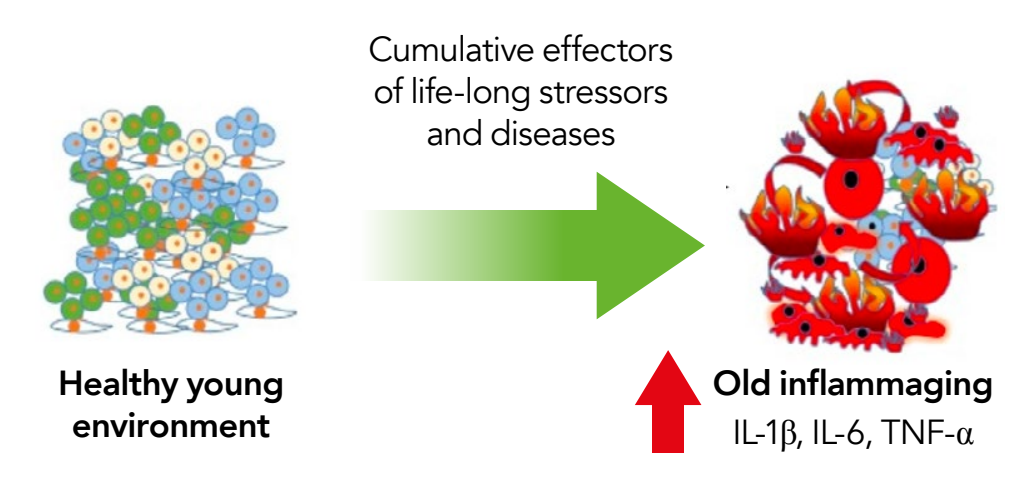
Inflammaging refers to the chronic, low-grade inflammation that characterises ageing. In young
adults, tissue microenvironment is healthy whereas cumulative insults by stressors and diseases over

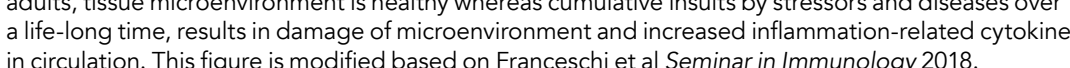

in 465 people between 21-88 years over of immune cells that includes $T$, B and NK cells, the group found that telomere length decreased as the participants aged. Interestingly, the rate of change the different functions they fulfi. each division, cells do have a tool they can use to lengthen their telomeres known as telomerase. The levels of this enzyme are usually tightly controlled, probably dus to the risk of cancer. The of the variation seen in $T$ cell telomere among $T$ cells, $B$ cells and monocy

Despite the telomere shortening with 
different levels of telomerase activity in cells, in combination with other factors and physiogical features such as blood glucose level.

Notable was the fact that, as with the group's 2017 study, the differences observed varied substantially between participants. Most noticeably, age-related trajectories of telomere attrition, elevated

INDIVIDUALIZED AGE-ASSOCIATED CHANGES IN IMMUNE SYSTEM YOUNG

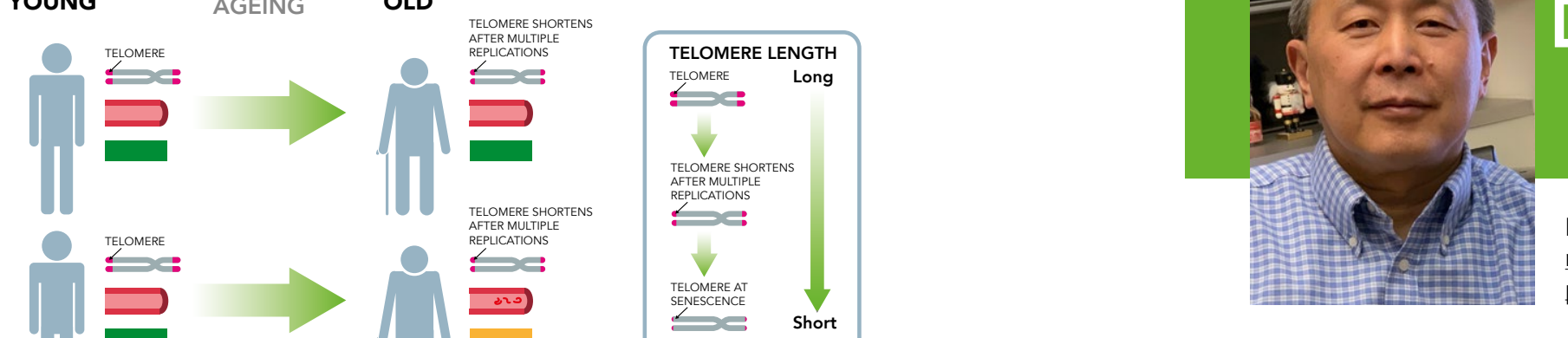
Behind the Research Dr Nan-ping Weng

E: Wengn@mail.nih.gov T: $+14105588341 \quad$ W: https://irp.nih.gov/pi/nan-ping-weng W: www nia.nih.gov/research/labs//mbil/ymphocyte-differentiation-section W: www.researchgate.net/ profile/Nan-ping Weng circulating inflammatory cytokines, and anti-CMV IgG are independent, and that ageing individuals do not show a uniform pattern of change in these variables

(Figure 3). Highlighting the importance of studying changes in individuals, rather groups together.

\section{EXPLAINING IMMUNE}

\section{SYSTEM DECLINE}

dimmune cells

be a link between the length of a person's

telomeres and the ability of their immune

system to defend againstinfection?

To tackle this question DrWeng and colleagues performed a study which was published in 2015 in The Journal of Infectious Diseases. In the paper, the group use a group of 22 healthy, older individuals, that had immune cells with either particularly short or long telomeres and determined the strength of their

immune response to an influenza vaccine.

As the body mounts an immune response to an invading virus such as influenza Bcells prod stick to the virus, rendering it unable to infect cells. Antibodies are highly specific so they must be generated for each

new strain of influenza virus that a person

encounters. As the vaccine contains

an inactive influenza virus, the researchers could measure the levels of influenza antibodies produced after participants received the shot and use it to determine the strength of their immune response. DrWeng's group were interested to see that those with a strong antibody response to influenza infection, had

\section{Research Objectives}

Dr Nan-ping Weng's research is focused on understanding the mechanism of age-related changes in immune repertoires, and naive and memory Tcellm function TCR human Longitudinal Study (BLSA) and mouse models.

\section{Detail}

\section{Bayview Blvd}

Baltimore,

USA
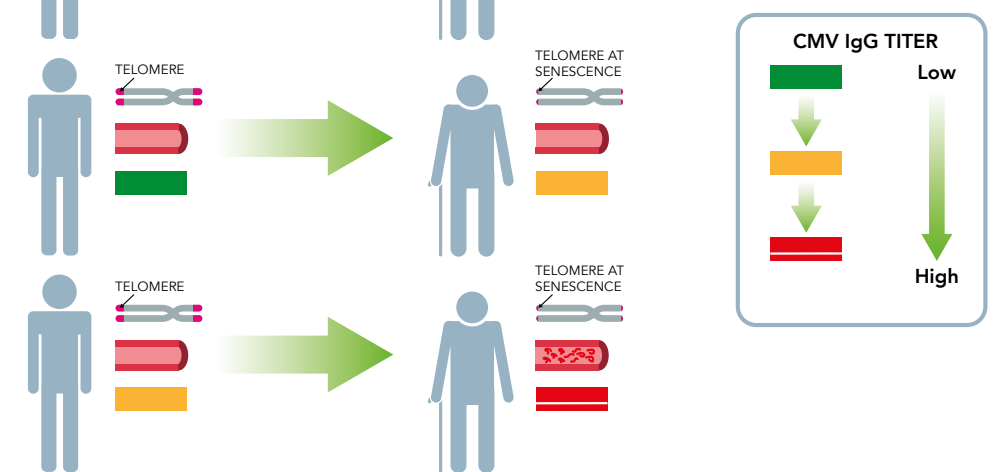

Ageing ofimmune system measured by three independent biomarkers: telomere length, inflammation-

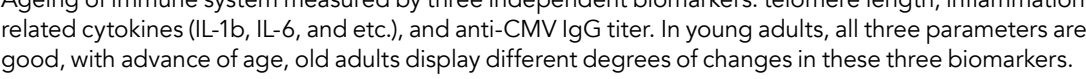
longer B cell telomeres than those with a weak response. They also found that $C D 8^{+} T$ cells also positively correlated with their ability to undergo cell divisions. This raises the possibility that shortening of telomeres in immune cells could contribute to the decline in a person's ability to fight off infection as they age.

\section{THE FUTURE}

The changes that occur during ageing and how these result in declining health, is an area of interest for society and scientists alike. By tracking changes in subjects over time, researchers such

When looking at Dr Weng's studies, one thing becomes clear, the way in which individuals age varies greatly. as DrWeng are helping to shed light on these questions.

When looking at Dr Weng's studies one thing becomes clear, the way in heterogeneity highlights the importance of his approach; if we simply average together groups of participants we may lose important information in the noise.

A proper understanding of the processes that underlie ageing is crucia if we are to improve our health in old age. This includes bolstering our ability to fight off infection as we get older and to enhance the function of the immune system when it gets "old", such as in influenza infection. We, therefore, awat with great anticipation. the case of reduced ability to fight off
Bio

Dr Weng received his MD from Fudan University Shanghai Medical College (former Shanghai First Medical College), China and PhD in Immunology from Baylor College of Medicine. He obtained his postdoctoral training at NC $\mathrm{NIH}$. He joined the National hstrtute on Aging (NIA) as a tenure-track investigator and now is a tenured senior investigator at the Laboratory of Molecular Biology and Immunology, NIA.

\section{Funding}

supported entirely by the Intramural Research Program of the NIH, National Institute on Aging.

\section{Collaborators}

- Luigi Ferrucci

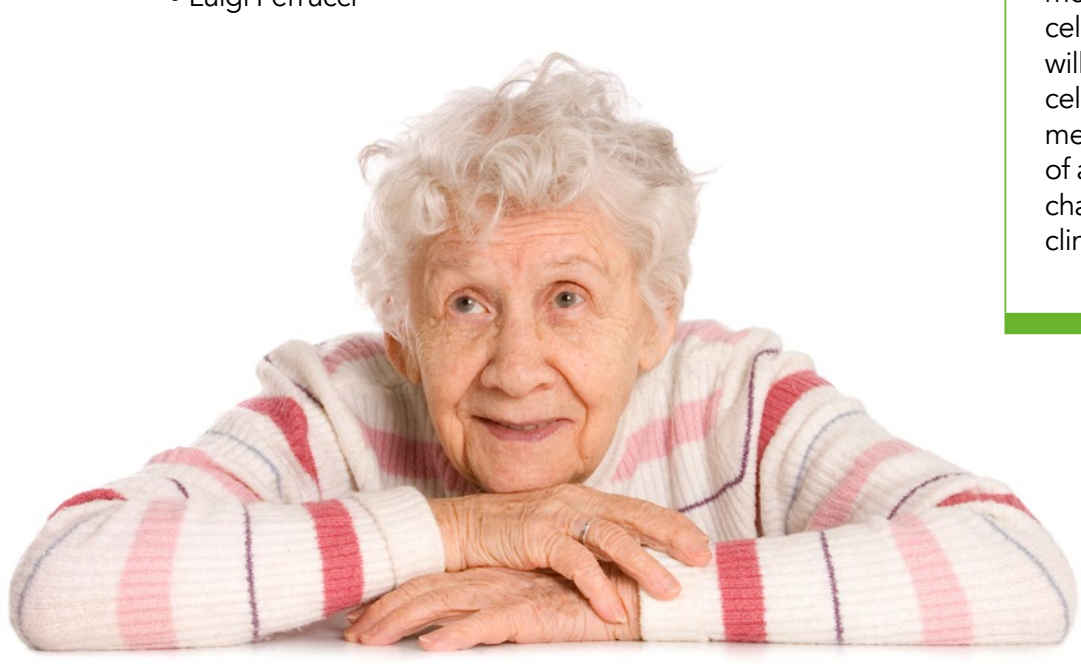

\section{References}

Lin Y, Damjanovic A, Metter EJ, Nguyen $H$, Truong T, Najarro K, Morris C, Longo DL, Zhan M, Ferrucci, Hodes RJ6, Weng in vivo is co-ordinated with changes in telomerase activity composition of lymphocyte subsets and health condition Clin Sci (Lond), 128(6):367-77. doi: 10.1042/CS20140481.

Lin, Y., Kim, J., Metter, E. J., Nguyen, H., Truong, T., Lustig, A.. Ferrucci, Weng, N. P. (2016). 'Changes in blood lymphocyte of cytokines/cytokine receptors'. Immunity \& Ageing: I \& A. 13,24 .

Lustig A, Liu HB, Metter EJ, An Y, Swaby MA, Elango P, Ferrucci L, Hodes RJ, Weng NP. (2017). 'Telomere Shortening, Inflammatory Cytokines, and Anti-Cytomegalovirus Antibody .

Najarro , Nguyen H1, Chen G, Xu M, Alcorta S, Yao X, Zukley L, Metter EJ, Truong T, Lin Y, Li H, Oelke M, Xu X, Ling SM, Longo DL, Schneck J, Leng S, Ferrucci L, Weng NP. (2015). Telomere Length as an Indicator of the Robustness of B-and T-Cell Response to In lnenza in Older Adult'. $J$ in

\section{Personal Response}

\section{What future studies do you have planned?}

II We are currently focusing on unravelling the underlying mechanisms of lymphocyte ageing from single cell to will be able to determine cellular ageing at the individual cell level and to apply the multi-parameters approach to of an individual allow us to determine general and specific changes in lymphocytes and to develop new tools for inical applications in precision medicine. 